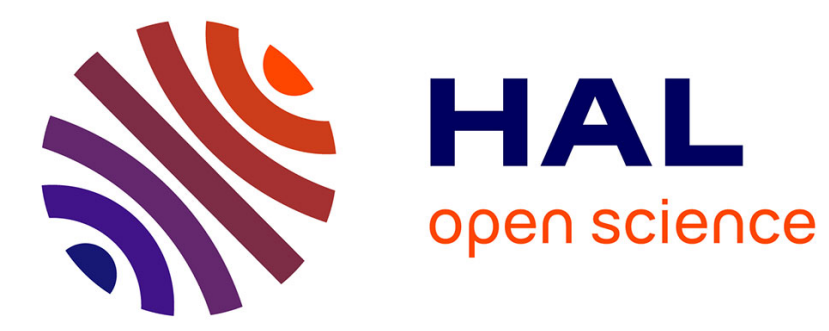

\title{
Performance Study of Cooperative Non-Orthogonal Multiple Access with Energy Harvesting
} Jiaoda Li, Chi Wan Sung, Chung Shue Chen

\section{To cite this version:}

Jiaoda Li, Chi Wan Sung, Chung Shue Chen. Performance Study of Cooperative Non-Orthogonal Multiple Access with Energy Harvesting. International Conference on Communication Engineering and Technology, Apr 2019, Nagoya, Japan. hal-01959158

\section{HAL Id: hal-01959158 \\ https://hal.inria.fr/hal-01959158}

Submitted on 18 Dec 2018

HAL is a multi-disciplinary open access archive for the deposit and dissemination of scientific research documents, whether they are published or not. The documents may come from teaching and research institutions in France or abroad, or from public or private research centers.
L'archive ouverte pluridisciplinaire HAL, est destinée au dépôt et à la diffusion de documents scientifiques de niveau recherche, publiés ou non, émanant des établissements d'enseignement et de recherche français ou étrangers, des laboratoires publics ou privés. 


\title{
Performance Study of Cooperative Non-Orthogonal Multiple Access with Energy Harvesting
}

\author{
Jiaoda $\mathrm{Li}^{*}$, Chi Wan Sung*, and Chung Shue Chen ${ }^{\dagger}$ \\ ${ }^{*}$ City University of Hong Kong, Tat Chee Avenue, Kowloon, Hong Kong SAR \\ ${ }^{\dagger}$ Nokia Bell Labs, Paris-Saclay, 91620 Nozay, France \\ Email: jiaodali2-c@my.cityu.edu.hk, albert.sung@cityu.edu.hk \\ chung_shue.chen@nokia-bell-labs.com
}

\begin{abstract}
Non-Orthogonal Multiple Access (NOMA) is a candidate channel access technique for the future generation wireless communication systems. It exploits the power domain to enable simultaneous access for multiple users. In this paper, a NOMA system with a base station (BS) and two users is studied. A novel scheme where the near user (UE 1) harvests energy from the signals sent from BS and relays the previously decoded message of the far user (UE 2) is proposed. It includes two common energy harvesting schemes, namely, power splitting and time switching, as special cases. The performance is evaluated by the achievable data rate of UE 2 under different requirements on the data rate of UE 1. Numerical simulations and analysis reveal that when the channel conditions between BS and UE 1, and between UE 1 and UE 2 are good, while that between BS and UE 2 is bad, the proposed scheme works the best and has a significant gain over the conventional NOMA scheme without cooperation. In addition, for the sake of simplicity, the power splitting scheme can be used to replace the generalized scheme for energy extraction without jeopardizing the performance gain much.
\end{abstract}

Index Terms-Non-Orthogonal Multiple Access (NOMA), cooperative NOMA, energy harvesting

\section{INTRODUCTION}

With the development of the Internet of Things (IoT), big data and many other new technologies, the next generation cellular communication systems are seeing enormous increase of connected devices. As a consequence, it poses a big challenge for the limited radio resources to cater for the huge number of users. Non-Orthogonal Multiple Access (NOMA) is one of the promising techniques to overcome the problem of resource scarcity.

In contrast with orthogonal multiple access schemes, NOMA supports simultaneous connections of multiple users with the same channel, and has the advantages of high spectral efficiency, massive connectivity, low latency, and high user fairness. For example, cooperative NOMA was proposed in [1] to take full advantage of the prior information that users with better channel conditions have about other users' messages.

On the other hand, with the popularization of IoT, there are envisioned to be many power-constrained nodes, which are hard to charge in conventional ways. It will be difficult to maintain their lifetime if they are required to transmit

This work was supported by the Research Grants Council, University Grants Committee, Hong Kong Special Administrative Region, China, under Project No. CityU 11216416. A part of the work was carried out at LINCS (www.lincs.fr) and during the internship of Jiaoda Li at Nokia Bell Labs. signals to far users by using significant transmission power. Therefore, energy harvesting has attracted much attention from the research community. Two practical designs, namely, time switching and power splitting, have been put forward in [2] for simultaneous power and information transmission. These two schemes have been widely accepted and used. For example, the power splitting scheme was applied to cooperative NOMA in [3]. It was found that simultaneous wireless information and power transfer (SWIPT) will not jeopardize NOMA's diversity gain, and the benefit of user selection based on node locations was demonstrated. In addition, a generalized scheme combining time switching and power splitting was proposed in [4].

Note that the work in [3] focused on system-level user pairing and its effect on system throughput and user outage probability, but did not consider optimization on the energy harvesting scheme. The work in [4] came up with a possible improvement by generalizing the time switching and power splitting schemes. Although it is shown that the generalized scheme can produce a larger achievable rate region than the two original schemes when the energy is applied to successive interference cancellation (SIC), whether the generalized scheme still outperforms the other two when it is used to generate energy for cooperative transmission still remains unknown. Therefore, a scheme where the generalized energy harvesting scheme is applied to a cooperative NOMA system is proposed. In this work, we have the following new findings and the major contributions are summarized as follows:

- The maximal data rate of the far user in a cooperative NOMA system with energy harvesting is derived, and it is found that the proposed scheme generally outperforms the conventional NOMA without cooperation. On the other hand, when the target data rate of the near user becomes large, the proposed scheme falls back into the conventional NOMA.

- The most applicable scenario for the proposed scheme is presented. When the channel conditions between the base station and near user, and between near user and far user are good, while that between the base station and far user is bad, the gain compared to the conventional NOMA scheme is most significant.

- Lastly, the three SWIPT receiving schemes are com- 
pared. The performance difference between power splitting scheme and generalized scheme is negligible in most cases. Hence, in practical systems, power splitting can be adopted to simplify hardware design.

\section{SySTEM Model}

Fig. 1 shows the downlink NOMA system with two users, where UE 1 is the near user and UE 2 is the far user. The downlink transmission consists of two phases, namely direct transmission phase (Phase 1) and cooperative transmission phase (Phase 2). We normalize the total time length of direct transmission phase to 1 . As the name indicates, the BS directly transmits to UE 1 and UE 2. Phase 1 is made up of two time slots, lasting for $t$ and $1-t$, respectively. UE 1 harvests energy only in the first slot and receives both information and energy simultaneously in the second slot, while UE 2 just receives information during the whole phase. Therefore, during the first slot, the BS transmits signal of UE 2 only, while the superposition of UE 1's and UE 2's signals is transmitted in the second slot. Since UE 1 has already decoded UE 2's data before decoding its own through SIC during Phase 1, it then uses the energy scraped up to transmit the decoded data to UE 2 during cooperative transmission phase (Phase 2). We assume that there is an out-of-band channel to support this device-todevice (D2D) communication between UE 1 and UE 2, which is commonly called outband D2D [5]. The technical details are explained below.

\section{A. Phase 1: Direct Transmission}

The signal sent by the BS during Phase 1 can be generally denoted by

$$
\sqrt{P_{1}^{(1, j)}} x_{1}+\sqrt{P_{2}^{(1, j)}} x_{2},
$$

where $P_{i}^{(1, j)}$ is the power allocated to UE $i$ in $j^{\text {th }}$ time slot, and the superscript ' 1 ' indicates Phase 1 (similar subscripts and superscripts are applied to data rate $R$, signal-to-noise ratio or signal-to-noise-plus-interference ratio $\gamma$ too). The maximal transmitted power of the BS is $P_{T}$, and $x_{1}$ and $x_{2}$ are the messages for UE 1 and UE 2, respectively. Then UE $i$ will receive

$$
y_{i}^{(1, j)}=h_{i} \sum_{k \in\{1,2\}} \sqrt{P_{k}^{(1, j)}} x_{k}+n_{i},
$$

where $h_{i}$ denotes the channel link gain from the BS to UE $i$, and $n_{i}$ is the additive white Gaussian noise (AWGN) with zero mean and variance $\sigma^{2}$.

Since in the first slot, only signal of UE 2 is transmitted, we have $P_{1}^{(1,1)}=0$. Besides, we set

$$
P_{2}^{(1,1)} \leq P_{T} .
$$

According to the Shannon-Hartley theorem, the instantaneous achievable rate during this time slot, $R_{2}^{(1,1)}$, is bounded above as follows:

$$
R_{2}^{(1,1)} \leq \log _{2}\left(1+\frac{\left|h_{2}\right|^{2} P_{2}^{(1,1)}}{\sigma^{2}}\right) .
$$

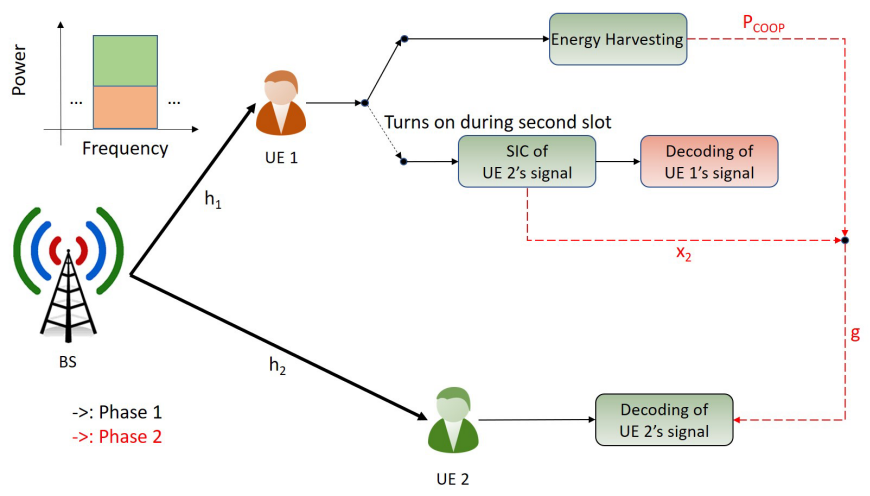

Fig. 1. System model

In the second slot, the superposition of both UE 1's and UE 2 's signals is transmitted. The power constraint becomes

$$
P_{1}^{(1,2)}+P_{2}^{(1,2)} \leq P_{T} .
$$

UE 1 divides the received power $P_{1}^{(1,2)}$ into two parts, one for energy harvesting, the other for signal decoding. We denote the proportion of first part by $\rho$, and second part by $1-\rho$.

Assumption 1: Interference can be totally eliminated by SIC, so the instantaneous achievable rate of UE 1 (the near user) within this time slot is given by

$$
R_{1}^{(1,2)} \leq \log _{2}\left(1+\frac{\left|h_{1}\right|^{2}(1-\rho) P_{1}^{(1,2)}}{\sigma^{2}}\right) .
$$

UE 2 (the far user) views signals of UE 1 as interference, so the received SINR at UE 2 is given by

$$
\gamma_{2}^{(1,2)}=\frac{\left|h_{2}\right|^{2} P_{2}^{(1,2)}}{\left|h_{2}\right|^{2} P_{1}^{(1,2)}+\sigma^{2}} .
$$

UE 2 does not decode its data at this stage. Instead, it will be decoded after combining with the signals received in Phase 2.

Assuming the energy harvesting efficiency is $\xi$, the energy harvested by UE 1 during Phase 1 is thus given by

$$
E_{H}=t \xi\left|h_{1}\right|^{2} P_{2}^{(1,1)}+(1-t) \rho \xi\left|h_{1}\right|^{2}\left(P_{1}^{(1,2)}+P_{2}^{(1,2)}\right) .
$$

\section{B. Phase 2: Cooperative Transmission}

During Phase 2, UE 1 relays the decoded message $x_{2}$ to UE 2. Then UE 2 will combine the signals received in the two phases using maximal ratio combining (MRC). In order to do that, we make Phase 2 as long as the second slot of Phase 1, which is $1-t$. (Note that one may consider other cooperative transmission and diversity combining schemes.) Hence, the total power consumption for cooperative transmission $P_{C}$ should satisfy

$$
(1-t) P_{C} \leq E_{H}
$$

UE 2 observes

$$
y_{2}^{(2)}=g \sqrt{P_{C}} x_{2}+n,
$$

where $g$ is the channel link gain from UE 1 to UE 2. The received SNR at UE 2 during Phase 2 is thus given by

$$
\gamma_{2}^{(2)}=\frac{|g|^{2} P_{C}}{\sigma^{2}} .
$$


After MRC, the obtained SINR is given by

$$
\gamma_{2}^{(M R C)}=\gamma_{2}^{(2)}+\gamma_{2}^{(1,2)}
$$

Therefore, the instantaneous achievable rate within this phase is bounded above as follows:

$$
R_{2}^{(M R C)} \leq \log _{2}\left(1+\gamma_{2}^{(M R C)}\right) .
$$

In addition, in order to meet Assumption 1, UE 1 should be able to decode UE 2's message successfully, i.e.,

$$
R_{2}^{(M R C)} \leq \log _{2}\left(1+\frac{\left|h_{1}\right|^{2}(1-\rho) P_{2}^{(1,2)}}{\left|h_{1}\right|^{2}(1-\rho) P_{1}^{(1,2)}+\sigma^{2}}\right)
$$

\section{PROBlem Formulation}

The performance of the proposed system is evaluated by the maximal $R_{2}$ under certain $R_{1}$, where $R_{1}$ and $R_{2}$ are the data rates of UE 1 and UE 2, respectively. Therefore, it is an optimization problem

$$
\begin{aligned}
\text { (P0) } \max _{t, \rho} & R_{2} \\
\text { s.t. } & R_{1}=r \\
& 0 \leq t \leq 1,0 \leq \rho \leq 1 \\
& (3)-(6),(9),(13),(14)
\end{aligned}
$$

where $r$ is the target average rate of UE 1 .

UE 1 receives signal only during the second slot of direct transmission phase, therefore

$$
R_{1}=(1-t) R_{1}^{(1,2)}
$$

UE 2's data reception comprises of two parts. The first part is from the BS during the first slot of Phase 1, and the second part is by the MRC of the data from the BS during the second slot of Phase 1, and from UE 1 during Phase 2. Consequently,

$$
R_{2}=t R_{2}^{(1,1)}+(1-t) R_{2}^{(M R C)}
$$

We let $r>0$. Otherwise, UE 1 will become a pure relay node. In this case, we can infer that both $t$ and $\rho$ cannot be 1: From (15b) and (16), we have

$$
r=(1-t) R_{1}^{(1,2)},
$$

so $t \neq 1$. And if $\rho=1$, according to (6), $R_{1}^{(1,2)}=0$, which implies $r=0$. Therefore, $\rho \neq 1$.

Note that in the objective function (17), $R_{2}^{(1,1)}$ is uncorrelated with $t$ and $R_{2}^{(M R C)}$, so in order to maximize $R_{2}$ we need to maximize $R_{2}^{(1,1)}$. Since $R_{2}^{(1,1)}$ is only constrained by (4), the equality in (4) should be satisfied. Similarly, the equalities in (13) and (14) should be satisfied since $R_{2}^{(M R C)}$ is constrained by both of them.

So, from (4), $R_{2}^{(1,1)}$ becomes an increasing function with respect to $P_{2}^{(1,1)}$, where $P_{2}^{(1,1)}$ is solely constrained by (3). This implies that the equality in (3) should be satisfied too. Note that the equalities of (5) and (9) can be proved similarly as well. For any given $P_{1}^{(1,2)}, P_{2}^{(1,2)}$ should be maximized. From (7), we can see that $\gamma_{2}^{(1,2)}$ is an increasing function of $P_{2}^{(1,2)}$. Moreover, the right hand sides of (9) and (14) both increase as $P_{2}^{(1,2)}$ increases, see (8) for (9). Besides, according to (11), $\gamma_{2}^{(2)}$ is an increasing function of $P_{C}$. Therefore, from (13) and (14), to maximize $R_{2}^{(M R C)}$, the equalities of (5) and (9) should be met.

When the equalities of aforementioned constraints are satisfied, the objective function (17) is a decreasing function of $P_{1}^{(1,2)}$, due to (7) and (14). On the other hand, $P_{1}^{(1,2)}$ is lower bounded by (6) for the SIC requirement. So, (6) should be satisfied with the equality. As a result, we conclude that the equality of constraints (3)-(6), (9), (13), (14) should be satisfied for the purpose of optimization.

From the expressions of (18) and (6), we write

$$
r=(1-t) \log _{2}\left(1+\frac{\left|h_{1}\right|^{2}(1-\rho) P_{1}^{(1,2)}}{\sigma^{2}}\right) .
$$

Since $P_{1}^{(1,2)}$ is upper bounded by $P_{T}$, see (5), we have

$$
r \leq(1-t) \log _{2}\left(1+\frac{\left|h_{1}\right|^{2}(1-\rho) P_{T}}{\sigma^{2}}\right) .
$$

Due to the above technical details, the problem (P0) can be thus rewritten as

$$
\begin{gathered}
\text { (P1) } \max _{t, \rho} \quad t \log _{2}\left(1+\frac{\left|h_{2}\right|^{2} P_{T}}{\sigma^{2}}\right)+(1-t) R_{2}^{(M R C)} \\
\text { s.t. } \quad(1-t) \log _{2}\left(1+\frac{\left|h_{1}\right|^{2}(1-\rho) P_{T}}{\sigma^{2}}\right) \geq r \\
0 \leq t<1,0 \leq \rho<1
\end{gathered}
$$

where

$$
\begin{gathered}
R_{2}^{(M R C)}=\min \{ \\
\log _{2}\left(1+\frac{\left|h_{2}\right|^{2} P_{2}^{(1,2)}}{\left|h_{2}\right|^{2} P_{1}^{(1,2)}+\sigma^{2}}+\frac{\xi\left|h_{1}\right|^{2}|g|^{2} P_{T}\left(\frac{t}{1-t}+\rho\right)}{\sigma^{2}}\right) \\
\left.\log _{2}\left(1+\frac{\left|h_{1}\right|^{2}(1-\rho) P_{2}^{(1,2)}}{\left|h_{1}\right|^{2}(1-\rho) P_{1}^{(1,2)}+\sigma^{2}}\right)\right\}, \\
P_{1}^{(1,2)}=\frac{\left(2^{\frac{r}{1-t}}-1\right) \sigma^{2}}{\left|h_{1}\right|^{2}(1-\rho)}
\end{gathered}
$$

and

$$
P_{1}^{(1,2)}+P_{2}^{(1,2)}=P_{T}
$$

Note that the problem (P1) can be solved by using an exhaustive search algorithm (Algorithm 1). Since there are only two variables ( $\rho$ and $t$ ) in the generalized energy harvesting scheme, in practice it is fast to find the optimal solution in the above problem. Note that for power splitting scheme and time switching scheme, we can set simply $t=0$ or $\rho=0$, respectively. 


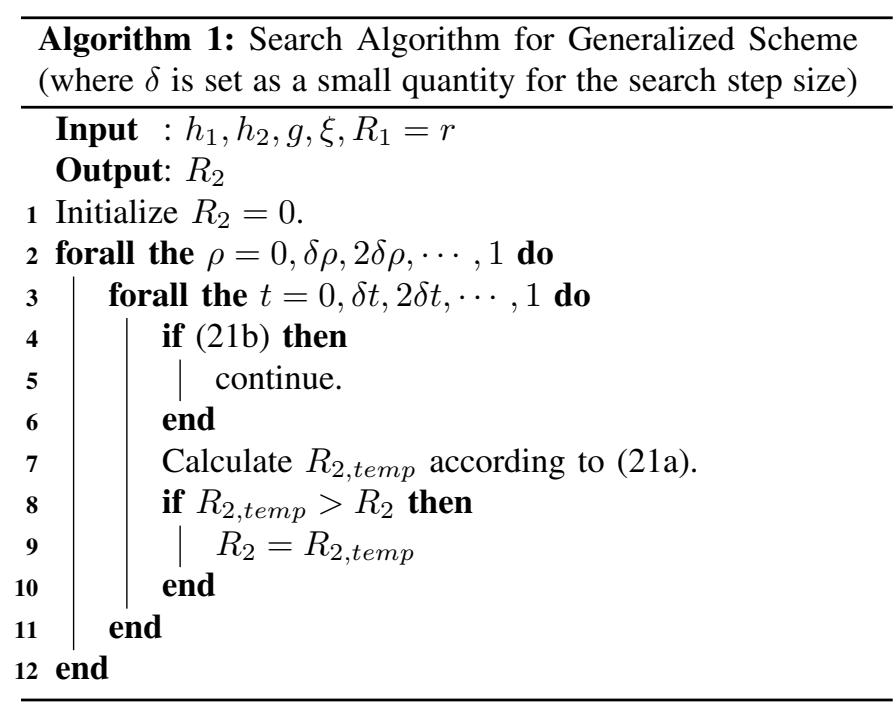

TABLE I

SYSTEM PARAMETERS

\begin{tabular}{|c|c|}
\hline Parameter & Value \\
\hline \hline Maximal transmission power of BS $P_{T}$ & $40 \mathrm{~W}$ \\
\hline Variance of AWGN $\sigma^{2}$ & $0.1 \mathrm{~mW}$ \\
\hline Channel link gain between BS and UE 1 & 0.01 \\
\hline Channel link gain between BS and UE 2 $\left|h_{2}\right|^{2}$ & 0.0001 \\
\hline Channel link gain between UE 1 and UE 2 $|g|^{2}$ & 0.01 \\
\hline Energy harvesting efficiency $\xi$ & 0.5 \\
\hline
\end{tabular}

\section{NUMERICAL RESULTS}

The numerical results are presented below and the system parameters [4] are listed in Table I.

We first study the achievable rate regions of the three energy harvesting schemes, namely, the generalized scheme, the power splitting scheme (i.e., $t=0$ ), and the time switching scheme (i.e., $\rho=0$ ). The conventional NOMA scheme, which has no cooperation phase (i.e., $\rho=t=0$ ), see below for the completeness, is also considered for comparison.

\section{A. Conventional NOMA}

Note that the total power budget is $P_{T}$. The power allocated to UE 1 and UE 2 are denoted as $P_{1}$ and $P_{2}$, respectively. We have

$$
\begin{gathered}
P_{1}+P_{2} \leq P_{T}, \\
R_{1} \leq \log _{2}\left(1+\frac{\left|h_{1}\right|^{2} P_{1}}{\sigma^{2}}\right), \\
R_{2} \leq \log _{2}\left(1+\frac{\left|h_{2}\right|^{2} P_{2}}{\left|h_{2}\right|^{2} P_{1}+\sigma^{2}}\right) .
\end{gathered}
$$

In order for UE 1 to implement SIC successfully,

$$
R_{2} \leq \log _{2}\left(1+\frac{\left|h_{1}\right|^{2} P_{2}}{\left|h_{1}\right|^{2} P_{1}+\sigma^{2}}\right) .
$$

Let

$$
f(x)=\frac{x P_{2}}{x P_{1}+\sigma^{2}}
$$

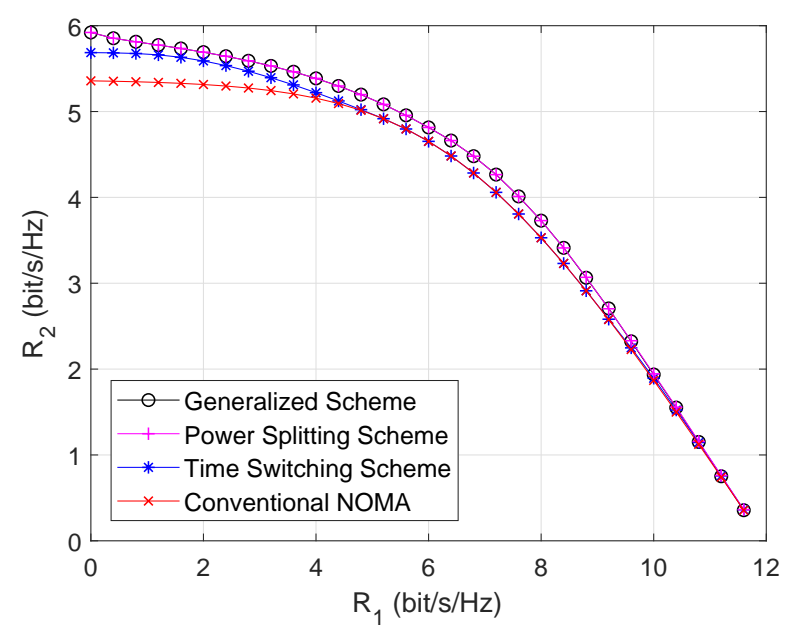

Fig. 2. Achievable rate regions of different schemes

Taking the first derivative, we get

$$
f^{\prime}(x)=\frac{P_{2} \sigma^{2}}{\left(x P_{1}+\sigma^{2}\right)^{2}}>0 .
$$

Therefore, $f(x)$ is an increasing function. Since UE 1 is closer to the BS than UE 2, it is generally reasonable to assume $\left|h_{1}\right|^{2}>\left|h_{2}\right|^{2}$, then

$$
f\left(\left|h_{1}\right|^{2}\right)>f\left(\left|h_{2}\right|^{2}\right)
$$

i.e.,

$$
\log _{2}\left(1+\frac{\left|h_{1}\right|^{2} P_{2}}{\left|h_{1}\right|^{2} P_{1}+\sigma^{2}}\right)>\log _{2}\left(1+\frac{\left|h_{2}\right|^{2} P_{2}}{\left|h_{2}\right|^{2} P_{1}+\sigma^{2}}\right) .
$$

Thus, $R_{2}$ is constrained by (27). The equalities in (25), (26) and (27) should also be satisfied for maximization. So we have

$$
R_{2}=\log _{2}\left(1+\frac{\left|h_{2}\right|^{2} P_{2}}{\left|h_{2}\right|^{2} P_{1}+\sigma^{2}}\right),
$$

where

$$
P_{1}=\frac{\left(2^{r}-1\right) \sigma^{2}}{\left|h_{1}\right|^{2}}
$$

and

$$
P_{1}+P_{2}=P_{T}
$$

\section{B. Achievable Rate Region and Gains}

Fig. 2 shows the result. The horizontal axis is target rate for UE $1, R_{2}$ is obtained by solving problem (P1) numerically for each $R_{1}(=r)$. As we can see, the cooperative schemes outperform conventional NOMA. We can see that the line of power splitting scheme basically coincides with the generalized scheme, and both of them are better than the time switching scheme. The improvement is more obvious when $R_{1}$ is small.

Note that when $t$ increases, although the harvested energy to be used in cooperative transmission is larger, the time length $1-t$ of cooperative transmission phase becomes shorter, and thus $R_{2}$ will not change much. As a result, the maximal $R_{2}$ would not be much different from the $R_{2}$ at $t=0$. So approximately, we can say $t=0$ produces the solution for time 


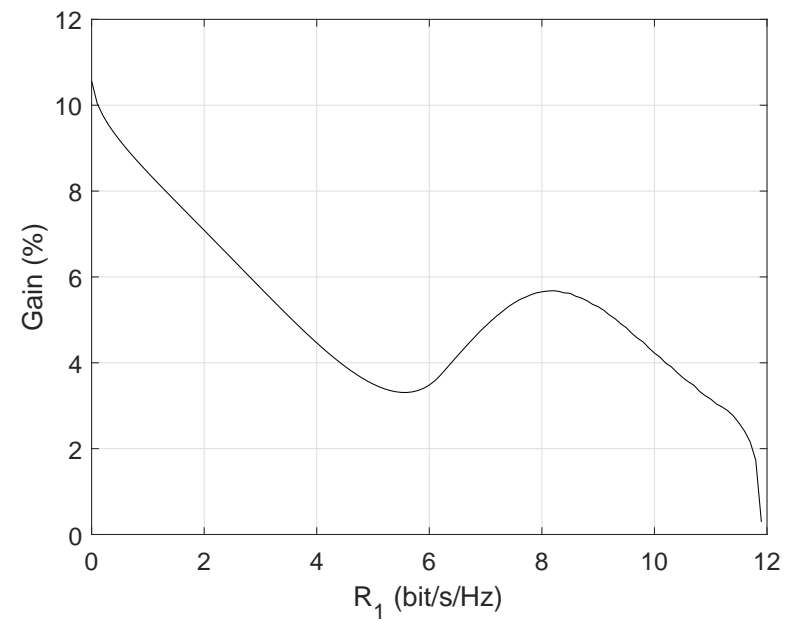

Fig. 3. Gain of the generalized scheme over conventional NOMA

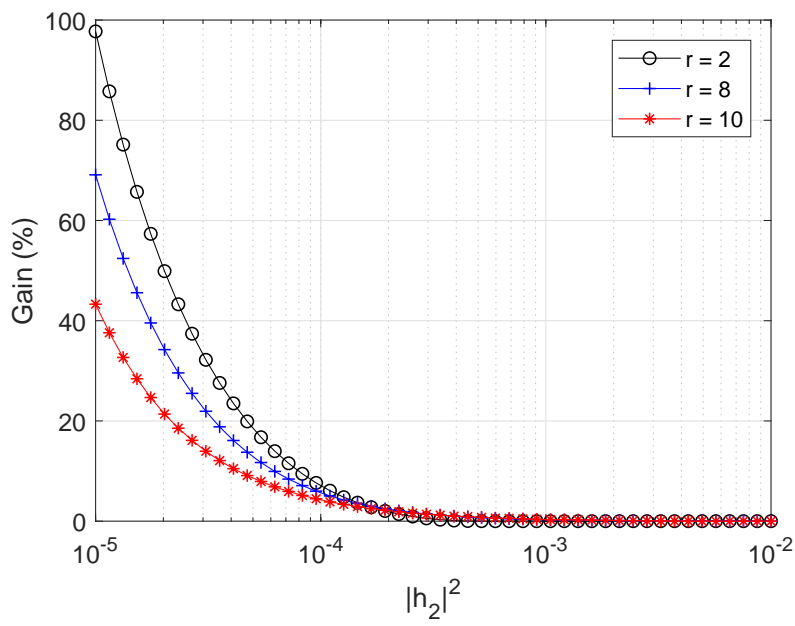

Fig. 4. Gain of the generalized scheme with respect to $\left|h_{2}\right|^{2}$

switching scheme. As the generalized scheme is nothing but a combination of time switching scheme and power splitting scheme, if $t=0$, the generalized scheme will become power splitting scheme. We can simply use power splitting scheme instead of the generalized scheme in practice, which can largely reduce the complexity.

In Fig. 3, we plot the performance gain in $R_{2}$ of the proposed scheme over conventional NOMA. Under this specification, the largest gain is around $10 \%$. And when $R_{1}$ is too large, there is no gain at all. From Fig. 3, we can see the gain does not decrease all the way when $R_{1}$ increases. Instead, it bounces up and down before going to zero. Target rate for UE 1 can be set to around 8 to achieve the local maximum, which is a good trade-off between $R_{1}$ and $R_{2}$, whereas $R_{1}$ in the leftmost part of the figure is too low although the gain on $R_{2}$ is high.

Next, we explore the relationship between the performance gain of the proposed scheme over conventional NOMA and the channel condition of UE 2 from BS. Fig. 4 shows the result. Intuitively, we know when the channel between the BS and UE 2 is totally blocked, the only way to reach UE 2 is by relaying through UE 1 . Wherefore, for the conventional NOMA without cooperative transmission, UE 2 cannot be reached when $\left|h_{2}\right|^{2}=0$. The proposed scheme becomes extremely important. In addition, when $\left|h_{2}\right|$ is as good as $\left|h_{1}\right|$ (and $|g|$ ), the cooperative transmission becomes less relevant. We can observe the above results from Fig. 4.

In practice, it often occurs that some devices are blocked by obstacles or under poor channel condition. One advantage of the proposed scheme is that other devices that are not being blocked can serve as relays to help the BS to reach those devices and reduce user outage probability. Note that when the above scenario does not happen, the energy harvesting scheme falls back into conventional NOMA, which means it will not cost unnecessary extra energy consumption and the data rate of UE 1 will not be jeopardized. In other words, the cooperative transmission and energy harvesting is turned on when necessary and off when not needed.

\section{CONCLUSION}

In this paper, the performance of cooperative NOMA with energy harvesting has been studied. The maximal data rate for the far user in a system adopting the proposed scheme has been proven to be larger than conventional NOMA. The proposed scheme has a larger gain when the channel condition between the BS and UE 2 is poor. On the other hand, when cooperative transmission is not suitable, the proposed scheme will simply degrade to conventional NOMA. Lastly, the different energy harvesting schemes have been compared. It is shown that power splitting has similar performance to the generalized scheme, but has simpler hardware design. Therefore, it is concluded that the reception quality of UE 2 can be enhanced if UE 1 relays the message to it, and no extra battery energy is consumed if UE 1 adopts the near user power-splitting energy harvesting scheme.

There are some future research possibilities. For instance, more realistic channel model can be used instead of assuming static condition. One may also consider the cancellation of signal during SIC cannot be complete. Besides, more sophisticated combining techniques rather than MRC can be used when UE 2 combines the signals from the BS and UE 2. Then the performance of time switching scheme is expected to be improved.

\section{REFERENCES}

[1] Z. Ding, M. Peng, and H. V. Poor, "Cooperative non-orthogonal multiple access in 5G systems," IEEE Wireless Commun. Lett., vol. 19, no. 8, pp. 1462-1465, Aug 2015.

[2] R. Zhang and C. K. Ho, "MIMO broadcasting for simultaneous wireless information and power transfer," IEEE Trans. Wireless Commun., vol. 12, no. 5, pp. 1989-2001, May 2013.

[3] Y. Liu, Z. Ding, M. Elkashlan, and H. V. Poor, "Cooperative nonorthogonal multiple access with simultaneous wireless information and power transfer,' IEEE J. Sel. Areas Commun., vol. 34, no. 4, pp. 938953, April 2016.

[4] J. Gong and X. Chen, "Achievable rate region of non-orthogonal multiple access systems with wireless powered decoder," IEEE J. Sel. Areas Commun., vol. 35, no. 12, pp. 2846-2859, Dec 2017.

[5] A. Asadi, Q. Wang, and V. Mancuso, "A survey on device-to-device communication in cellular networks," IEEE Commun. Surveys \& Tutorials, vol. 16, no. 4, pp. 1801-1819, Fourth Quarter 2014. 